PATIENT SAFETY

\author{
S.I. Moskowitz \\ W.J. Davros \\ M.E. Kelly \\ D. Fiorella
}

P.A. Rasmussen

T.J. Masaryk

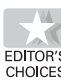

\title{
Cumulative Radiation Dose during Hospitalization for Aneurysmal Subarachnoid Hemorrhage
}

BACKGROUND AND PURPOSE: Radiation exposure from neurointerventional procedures and diagnostic neuroimaging can be substantial, with many recommendations offered to guide the interventionalist in the conscientious use of ionizing radiation. Patients presenting with an aneurysmal subarachnoid hemorrhage can undergo multiple imaging procedures during a prolonged hospital course. Therefore, we reviewed a cohort of such patients to identify the sources and quantify the cumulative radiation exposure seen during their hospitalization.

MATERIALS AND METHODS: We retrospectively reviewed a single-center experience with these patients to define the potential for short-term skin injury and long-term oncologic risk due to absorbed radiation dose and sources of ionizing radiation and their contribution to the cumulative absorbed dose to the cranial tissues.

RESULTS: We demonstrated that substantial cumulative doses can be seen, with $87 \%$ of the cumulative absorbed dose occurring during neurointerventional procedures and $7 \%$ from $\mathrm{CT}$. Mathematic modeling was performed identifying potential techniques to further reduce the cumulative radiation absorbed dose to these patients.

CONCLUSIONS: We conclude that repetitive irradiation during the care of patients with aneurysmal subarachnoid hemorrhage can result in significant cumulative doses and a variety of techniques can be applied to reduce this absorbed dose. Use of radiation for diagnostic and therapeutic purposes during prolonged procedures of patients with subarachnoid hemorrhage demands diligence throughout the hospitalization.

ABBREVIATIONS: CTA $=\mathrm{CT}$ angiography; $\mathrm{CTDI}=\mathrm{CT}$ dose index; DSA $=$ digital-subtraction angiography; ICRU = International Commission on Radiation Units and Measurements; MRI = MR imaging; $\mathrm{NS}=$ not significant

$\mathbf{A}^{\text {n }}$

$n$ understanding of the potential for short-term deterministic and long-term stochastic harm associated with the use of ionizing radiation for diagnostic and therapeutic purposes has been growing. Guidelines have been published by the US Food and Drug Administration International Commission on Radiologic Protection and the National Cancer Institute for monitoring operator and patient maximal doses and physician training to reduce the potential injury to patients and operators from radiation exposure. ${ }^{1-3}$ Embolization of cerebral aneurysms and arteriovenous malformations has been identified as a procedure that routinely exposes the patient and operator to high doses of radiation. ${ }^{4-15}$

Following an aneurysmal subarachnoid hemorrhage, patients are hospitalized for treatment of multiple neurologic and medical sequelae. Interventions that use ionizing radiation are integral to the continued care of these patients and include aneurysm embolization and angioplasty for vasospasm. Diagnostic imaging, including conventional angiography, CT, and plain $\mathrm{x}$-ray films, uses ionizing radiation as well in the management of neurologic and secondary medical diagnoses (eg, hydrocephalus, vasospasm, deep vein thrombo-

\section{Received April 29, 2008; accepted after revision March 9, 2010.}

From the Neurological Institute (S.I.M., P.A.R., T.J.M.) and Imaging Institute (W.J.D., T.J.M.), Cleveland Clinic Foundation, Cleveland, Ohio; Division of Neurosurgery (M.E.K.), Royal University Hospital, Saskatoon, Saskatchewan, Canada; and Department of Neurosurgery (D.F.), Stony Brook University Medical Center, Stony Brook, New York.

Please address correspondence to Shaye I. Moskowitz, MD, PhD, Section of Cerebrovascular and Endovascular Neurosurgery, Department of Neurosurgery, S80, Cleveland Clinic Foundation, Cleveland, OH, 44195; e-mail: moskows@ccf.org

DOI 10.3174/ajnr.A2132 ses), which can complicate the hospital course. Furthermore, repetitive imaging is frequently necessary.

The literature, which has reported the radiation doses given in a single cerebral interventional or diagnostic study, underestimates the cumulative craniocervical radiation and, therefore, the risk to patients during their hospitalization. We report a single-center experience with patients with aneurysmal subarachnoid hemorrhage, attempting to identify and quantify all sources and doses of craniocervical radiation, to better determine the cumulative doses these patients receive during the course of initial hospitalization. In addition, we use mathematic modeling to identify techniques to reduce the cumulative exposure.

\section{Materials and Methods}

\section{Study Design}

A retrospective chart review was performed with institutional review board approval, screening for aneurysmal subarachnoid hemorrhage treated in 2007. Medical records were reviewed for patient demographics, including length of stay, aneurysm number and maximum dimension, mode of treatment, Hunt and Hess grade, and Fisher score. Aneurysms with the largest diameter measuring $>10 \mathrm{~mm}$ were considered large. All cranial and peripheral imaging during the hospitalization by using ionizing radiation was identified, including fluoroscopic procedures, CT scans, and diagnostic x-ray imaging. All imaging was performed at the discretion of the neurosurgeon or neurointensivist as was clinically indicated. As a matter of routine practice, DSA or 3D rotational angiography was not performed. Conventional angiography was reserved for the initial diagnosis when the 
CTA was nondiagnostic and throughout the hospitalization when vasospasm was suspected or the aneurysm required reimaging, and $3 \mathrm{D}$ rotational angiography was used for unusually complex anatomy.

\section{Equipment and Radiation Dose}

All equipment is routinely monitored by a certified medical physicist with scheduled maintenance and annual measurements of radiation output and image quality. Phantoms used for system calibration used the standard CTDI. ${ }^{16}$ Fluoroscopic neurointerventional procedures were performed on a biplane Axiom Artis machine (Siemens, Erlangen, Germany). Filtration and collimation were routinely used as appropriate and individualized to each patient. DSA was performed at 2 frames per second, and fluoroscopy was pulsed at 30 pulses per second as per manufacturer recommendations for automatic programming. Cumulative air kerma was calculated by the software package installed on the equipment (Version VC12B, Siemens) and summed for both planes. To calculate skin absorbed dose, we multiplied this by 1.57 to convert to air kerma as per ICRU Report $47^{17}$ with the assumption of fluoroscopy and angiography as a wide energy spectrum beam with a tube voltage of $80 \mathrm{kV}$.

Skin absorbed dose was also collected separately for interventional procedures and diagnostic angiography. For each individual procedure, radiation absorbed doses for fluoroscopy and for angiographic runs were recorded separately. For patients whose aneurysms were treated surgically, an intraoperative angiogram was routinely obtained by using a standard single-plane C-arm Siremobil Iso-C scanner (Siemens) with automatic variable spectral filtration, variable kilovolt and milliampere settings based on patient transparency, and variable focal spot settings. Absorbed dose is not reported by this equipment; therefore, an estimated absorbed dose of $310 \mathrm{mGy}$ was considered. This was based on scheduled measurements of radiation exposure taken by the medical physics group at our institution for 3 angiographic runs of 2 images per second for 7 seconds and an additional 5 minutes of fluoroscopy time performed at 15 frames per second. Peripheral interventional procedures included venography for placement of Greenfield filters (6 patients), percutaneous gastrostomy tubes ( 4 procedures in 3 patients), modified barium swallow studies (twice in 1 patient), and central venous catheterization (indwelling catheter in 1 patient).

CT was performed on a multidetector Somatom Sensation 64-row scanner (Siemens), with sequential acquisition at a $20^{\circ}$ gantry tilt through the canthomeatal line. Measured absorbed skin dose for a single nonenhanced scan is $46.4 \mathrm{mGy}$, as measured with the previously described calibrated ion chamber and CTDI. CTA was performed on the same fixed scanners, by using a helical acquisition protocol with scanning from C2 through the vertex, and resulted in $46.4 \mathrm{mGy}$ of irradiation. CT perfusion was performed by using 2 images at the level of the basal ganglia, with 40 sections in repetitive acquisition for each of 2 adjacent sections at a $5-\mathrm{mm}$ distance. The measured absorbed dose for CT perfusion is $288.7 \mathrm{mGy}$. The measured absorbed dose for a CT of the sinuses is $22 \mathrm{mGy}$. Portable CT scanning of the brain, performed with a Ceretom NL-3000 (NeuroLogica, Danvers, Massachusetts) with a sequential acquisition at a zero-degree gantry tilt, resulted in $120 \mathrm{mGy}$ of irradiation.

To correct for irradiation resulting from noncervicocranial imaging, we reduced exposure by the distance to the irradiated field as follows ${ }^{18}$ : Cervical imaging resulted in $5 \%$ of the exposure to the head, and more remote imaging resulted in negligible exposure to the head. Therefore, other CT scans, including of the thoracolumbar spine, chest, abdomen, and pelvis, contributed a limited craniocervical ra-

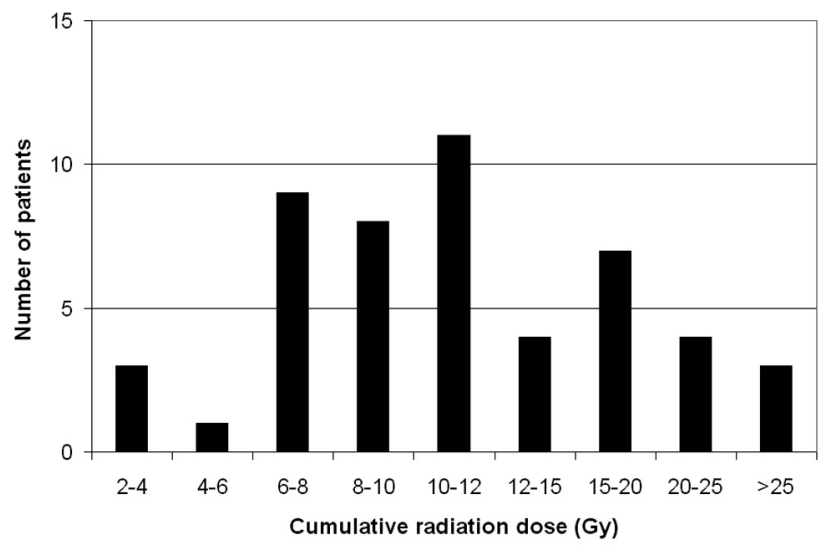

Fig 1. Histogram graphing the distribution of patients by cumulative radiation dose (in grays).

diation dose, with the exception of CTA of the cervical vasculature and CT of the cervical spine, which contributed $10 \mathrm{mGy}$ each.

Diagnostic x-ray imaging was performed with either digital portable or fixed equipment. Peripheral diagnostic imaging included chest or abdominal, skull, ankle, and cervical spine $\mathrm{x}$-rays. Skin doses from these studies were the following: cervical spine, $4 \mathrm{mGy}$; skull, 2.5 mGy. There was no direct radiation to the cranium from the other studies, and possible scatter was considered negligible.

\section{Statistical Analysis}

Data were analyzed by using a Student $t$ test to compare individual variables. A linear regression model was used for analysis of the length of hospitalization. A 1-way analysis of variance was performed for individual variables separately, including Hunt and Hess grade, Fisher score, and number of aneurysms. $P$ values $<.05$ were considered statistically significant.

\section{Results}

Fifty patients were identified harboring 70 aneurysms. The mean age was $56.1 \pm 14.4$ years (range, 29-86.9 years), and $68 \%$ were women. The mean Hunt and Hess grade was $2.9 \pm$ 1.0 and the mean Fisher score was $3.2 \pm 0.9$. The mean length of hospitalization was $17.0 \pm 11.1$ days (range, 2-64 days). The mean number of aneurysms per patient was $1.4 \pm 0.8$ (maximum, 4), and the mean size of the index aneurysm was $6.7 \pm 4.1 \mathrm{~mm}$ (range, $2-19 \mathrm{~mm}$ ). The treatment technique for the 50 index ruptured aneurysms was predominantly endovascular; 45 aneurysms were coiled, 5 were clipped, and 1 required both. Of the 20 nonindex aneurysms, 5 were coiled, 4 were clipped, and 11 were left untreated during the initial hospitalization.

The mean number of neurointerventional procedures performed was $1.2 \pm 0.7$. The mean number of CT scans obtained was $6.9 \pm 5.0$ (portable scans, $4.0 \pm 4.1$; fixed-equipment scans, $2.9 \pm 1.6$ ). The mean number of CTAs was $1.5 \pm 0.9$, and the mean number of CT perfusion scans was $0.6 \pm 0.9$. The mean number of chest $\mathrm{x}$-rays was $12.0 \pm 12.5$.

The mean cumulative radiation dose given to the cranium during the course of hospitalization was 12.8 $\pm 7.7 \mathrm{~Gy}$ (range, 2.4-36.1 Gy). The distribution of patients is graphed in Fig 1. The contribution from fluoroscopy and angiographic runs for interventional and diagnostic procedures, CT scans, and plain $\mathrm{x}$-ray imaging is graphed in Fig 2, demonstrating that the pre- 


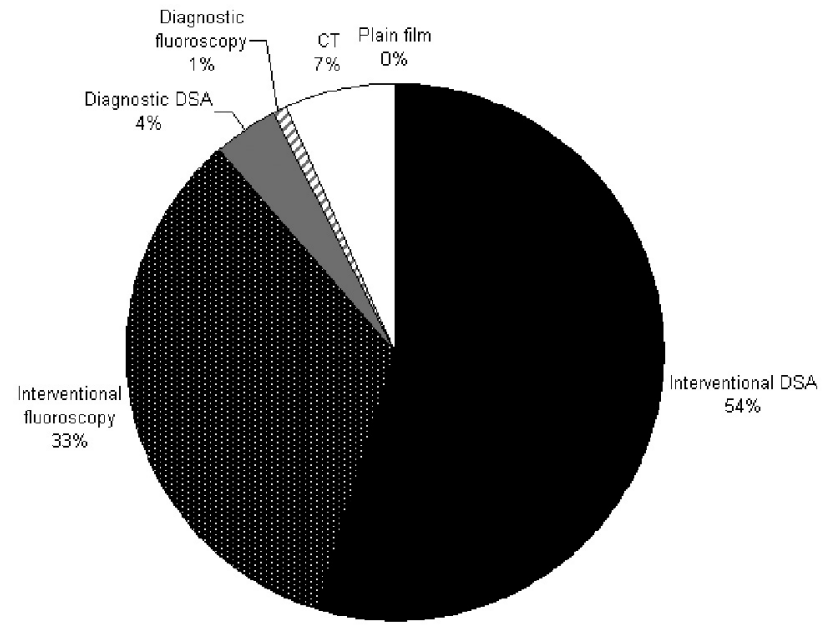

Fig 2. Contribution of various modes of imaging to the cumulative radiation doses. Interventional and diagnostic procedures are divided by angiographic runs and fluoroscopy.

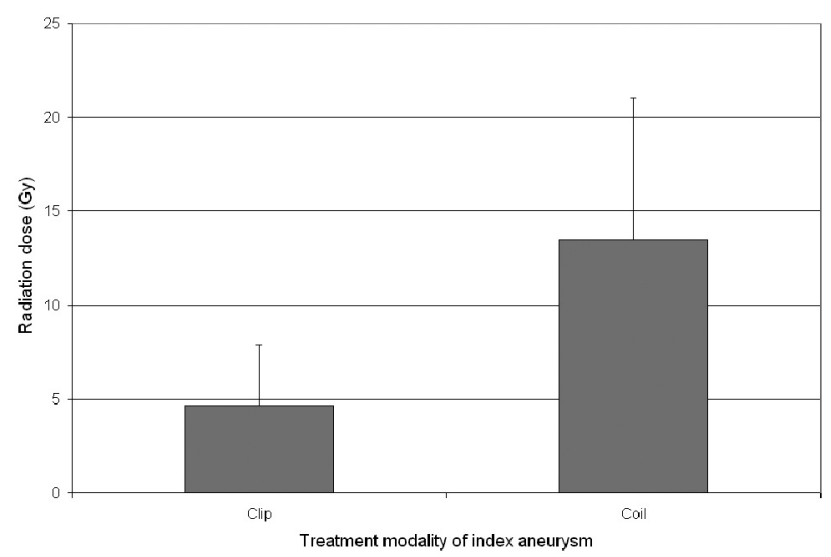

Fig 3. Comparison of the cumulative radiation doses between patients treated with either surgical clipping or coil embolization of the index aneurysm.

dominant exposure is in DSA runs during interventional procedures and that there is no substantial contribution from diagnostic $x$-ray imaging. The dose contributed by the coiling of the index aneurysm contributed a large portion of the total dose. For the 46 index aneurysms treated endovascularly, the mean procedural radiation dose was $10.4 \pm 4.2$ Gy (range, 3.9-20.3 Gy). This comprised the mean dose from fluoroscopy of 3.9 Gy and from DSA of 6.4 Gy.

Patients treated with coiling of the index aneurysm had a higher cumulative radiation dose (13.5 $\pm 7.5 \mathrm{~Gy})$ compared with those treated with clipping $(4.6 \pm 3.2$ Gy) (Fig 3; $P=$ .025). The Hunt and Hess grade, Fisher score, number of aneurysms, size of aneurysms (Fig 4; $P=$ NS for each variable individually), and length of hospitalization did not correlate with the cumulative cranial radiation dose (Fig $5 ; R=0.43$, $P=$ NS).

\section{Case Illustration}

The patient was a 55-year-old healthy man who presented with acute headache from a subarachnoid hemorrhage. He had a Hunt and Hess grade 3 and Fisher score 3 from a ruptured 3-mm anterior communicating artery aneurysm identified on CTA. The aneurysm was coiled on the subsequent day without difficulty (total absorbed dose, 12,439 mGy). He un-

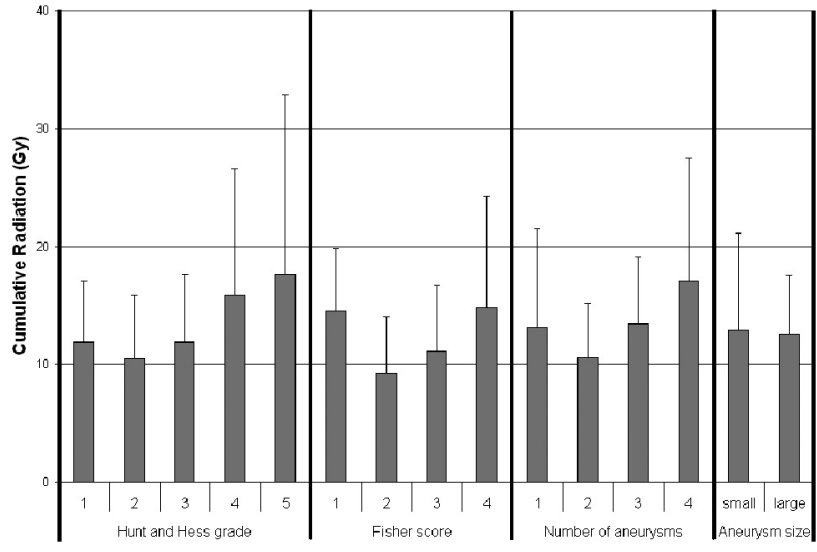

Fig 4. Comparison of the cumulative radiation as divided by Hunt and Hess grades, Fisher scores, number of cerebral aneurysms identified, or aneurysm size. Aneurysms with the largest diameter measuring $>10 \mathrm{~mm}$ are considered large.

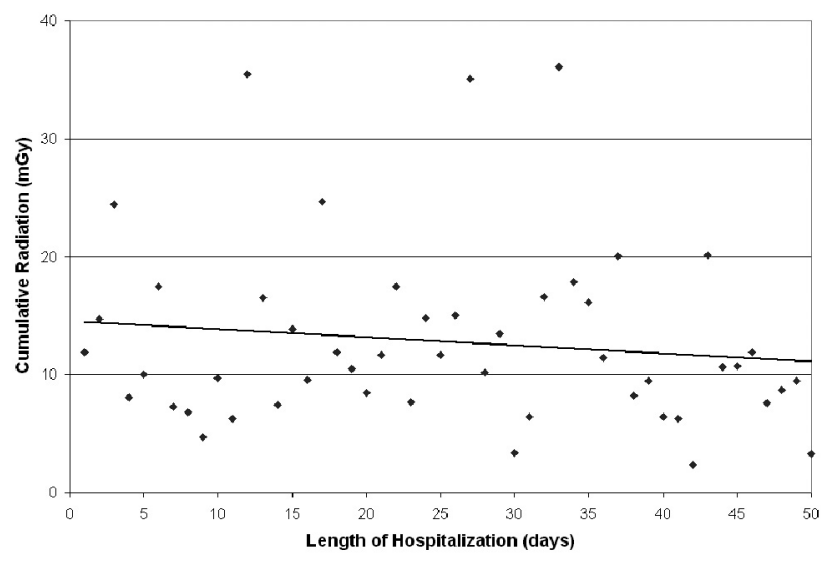

Fig 5. Scatterplot of cumulative radiation dose by length of hospitalization. A linear regression curve is best fit to the data $(R=0.45, P=\mathrm{NS})$.

derwent intracranial pressure and tissue oxygenation monitoring as well as external ventricular drainage. Serial transcranial sonography was routinely performed to survey for vasospasm. His condition deteriorated from medically refractory vasospasm and required 3 neurointerventional procedures (total absorbed doses, 5360, 4815, and 9566 mGy). His hospital course was complicated with multiple ventricular catheter changes and an associated right frontal parenchymal hemorrhage. Through a 45-day hospitalization, he required the following: 29 CT scans (7 routine scans on fixed equipment, 21 on portable equipment, and 1 volumetric scan for operative stereotactic shunt placement), 2 cranial CTAs and 1 CT perfusion, 32 chest and 6 abdominal x-rays, 1 shunt series, and 1 fluoroscopic placement of a percutaneous gastrostomy. He was ultimately discharged in poor neurologic condition. His cumulative skin entry dose was $35.5 \mathrm{~Gy}$.

\section{Discussion}

Ionizing radiation is a critical component of the diagnosis and treatment of cerebrovascular disease. With the increasing use of endovascular techniques and the utility, speed, and quality of CT technology, management of the patient with complex subarachnoid hemorrhage has evolved to routine use of these modalities. The repetitive imaging, however, carries the risk of radiation exposure to both the patient and physician. This 
recognized radiation risk has prompted multiple medical committees to regulate and monitor equipment and recommend techniques to reduce the exposure. ${ }^{1-3}$ While studies have identified cerebral embolization as a high-radiation exposure procedure, ${ }^{4-11,19}$ the cumulative exposure to the patient from repetitive procedures and imaging studies within a single hospitalization has not been documented. We demonstrate that the radiation exposure of the index procedure substantially underestimates the total radiation given through a full hospitalization.

The greatest contribution to the radiation exposure came during neurointerventional procedures composing $87 \%$ of the total exposure, of which 54\% was during DSA runs and 33\% was during fluoroscopy during any endovascular procedure. Considering the index treatment only, dose from DSA runs contributed $46.2 \%$, while fluoroscopy contributed $28.3 \%$. This finding suggests that a significant contributor to the exposure is the index procedure. However, the contribution from secondary procedures cannot be dismissed as insignificant. Despite the frequent use of CT scanning, this contributed only $7 \%$ of the total radiation. Peripheral imaging and diagnostic $x$-ray films had no meaningful contribution, largely because doses used for this imaging technique are markedly lower than those regularly seen during neurointerventional procedures. Additionally, the head is not routinely in the imaged field during systemic imaging, and the cranial exposure from scatter is insignificant. Although it is logical to focus on the initial catheter-based procedures as the principle exposure source, subsequent procedures and other imaging modalities are significant contributors as well.

\section{Risk of Injury}

The exact risk to the patient associated with radiation exposure remains to be clarified. The deterministic effects of the radiation, ones that are dose-dependent, are more likely to be seen with increasing exposure and are more clearly understood. ${ }^{20}$ Our data suggest that the patient with aneurysmal subarachnoid hemorrhage is at particular risk because of the high doses received during a short period of time. The stochastic effects of the radiation, ones that are dose-independent, are less understood compared with the higher doses seen for these patients. Long-term outcome studies have not explored the effects of radiation in the patient with subarachnoid hemorrhage, and reports of short-term complications of radiation exposure are limited. ${ }^{6,21-23}$ Because the essential question that underlies this and other studies of radiation exposure for this patient group is one of short- and long-term risk, better controlled longitudinal studies are needed to follow patients for the development of skin, lens, thyroid, and cerebral injury or neoplasia.

The doses described here are comparable with doses known to result in severe late secondary skin injury and delayed neoplasia. ${ }^{20}$ These clinical complications have not been seen in follow-up to date, and the minor side effects, including epilation and erythema, were not routinely documented. Significant injury may not have been witnessed in this population despite the high doses because the topographic dose distribution may not be accurately reflected by the total dose reported by the equipment. Furthermore, the actual dose may not be correctly represented by that reported by the equipment. A complementary longitudinal study will be important in specifically determining whether these patients develop any delayed radiation-induced injury. Nonetheless, the data suggest that special consideration be given to the patient with aneurysmal subarachnoid hemorrhage throughout the hospitalization, particularly because of the potential for cumulative exposures from repetitive studies.

\section{Distribution of Dose}

Fluoroscopy has a peak exposure at the skin next to the radiation source, and in biplanar fluoroscopy, the dose is maximally distributed at 2 points, typically posterior and left lateral (to minimize operator exposure). CT scanning evenly distributes the irradiation, while CT perfusion maximally irradiates a small cross-section. We estimated the exposure by combining the estimated entry-skin dose for each procedure and imaging study because it is most likely to reflect the cumulative radiation to the cranium. This is an understood limitation, in that the cumulative and effective doses received will overestimate exposure, particularly if the radiation-sensitive tissue is not directly in the field of the fluoroscope.

Reporting radiation dose for complex exposures, like fluoroscopy, is difficult because multiple metrics are available, including fluoroscopy time, cumulative dose, dose-area product, and entry-skin dose. The ideal measure is one that predicts actual injury and risk. While the best estimate may be entryskin dose, ${ }^{24}$ the cranium receives a nonuniform distribution of irradiation, making estimation of radiation exposure difficult. Entry-skin-dose measurements are possible with use of thermoluminescent detectors on the skin at the point of maximal radiation. This technique, however, does not afford an immediate on-line measure and is not universally available; these effects limit its utility. Additionally, use of thermoluminescent detectors assumes placement at the exact point of maximal dose, which is not known a priori.

Furthermore, patterns of irradiation are complex and vary among patients as well as procedures; these patterns prevent an accurate risk profile for each set of procedures and individual patients. For example, in the case of the lens, the radiation source during fluoroscopy is usually posterior to the head. Because entry doses are substantially higher than the exit doses, irradiation of the lens is overestimated by simple tissueweighting of the total dose reported by the fluoroscopy equipment. Nonetheless, the estimation of cumulative doses allows an appreciation of the potential exposure accumulated during a brief hospitalization.

Doses from $<1$ to $>6$ Gy have been reported for an aneurysm coiling. ${ }^{4-11,19}$ However, the doses received from individual embolizations in our patient group were higher, with a mean dose during treatment of the index aneurysm of $12.8 \mathrm{~Gy}$. This high dose may be a reflection of the complexity of the aneurysms referred to our quaternary care institution, as well as from the use of biplanar fluoroscopy, as is standard during coiling of aneurysms. Additionally, treatment in a teaching institution inevitably results in extra dose from biplanar use when single plane imaging would be sufficient, filming through the venous phases of every run, extra runs between coil deployments, and so forth.

Another potential source of high exposure is the misunderstanding of the doses reported by the angiography equipment. 


\begin{tabular}{lcccc}
\hline \multicolumn{5}{l}{ Radiation-reduction techniques (in grays) } \\
\hline \multicolumn{5}{l}{$\mathbf{a}^{\circ}$} \\
& Mean & $\begin{array}{c}\text { } \\
\text { Reduction }\end{array}$ & Minimum & Maximum \\
\hline Unmodified & $12.8 \pm 7.7$ & & 2.4 & 36.1 \\
Fixed replacing portable CT & $12.5 \pm 7.5$ & 2.3 & 2.2 & 35.3 \\
Low-dose portable CT & $12.6 \pm 7.5$ & 1.9 & 2.2 & 35.5 \\
CTA replacing DSA & $12.2 \pm 7.6$ & 4.9 & 0.3 & 35.5 \\
MRI replacing CT & $12.0 \pm 7.3$ & 6.6 & 0.3 & 34.2 \\
Lower fluoroscopy rate & $11.3 \pm 6.8$ & 11.6 & 2.4 & 32.7 \\
3.6-2.7 $\mu$ Gy per frame & $11.0 \pm 6.5$ & 13.8 & 2.4 & 31.1 \\
3.6-1.8 $\mu$ Gy per frame & $9.1 \pm 5.3$ & 29.1 & 2.3 & 26.7 \\
All above & $6.4 \pm 4.0$ & 56.6 & 0.0 & 18.7 \\
\hline
\end{tabular}

a Calculated dose based on individual specific modifications of imaging techniques as described in the text. Dose was calculated for each patient and individual study and reported for the collective group.

Air kerma was reported by our equipment while skin-entry dose may be more important to monitor. Because the air kerma does not account for backscatter within the tissue itself, the dose is approximately increased by 1.4 -fold to 1.7 -fold (ICRU Report 47, Table A.3, page 25, 1992) depending on the kilovolt and energy spectrum of the beam. Understanding what is reported will lead to better patient monitoring.

The doses we identified are comparable with ranges of exposures known to correlate with mild-to-moderate skin injury, published by the US Food and Drug Administration ${ }^{1}$ and in the literature. ${ }^{20}$ However, the cumulative exposures we demonstrate are substantially higher. The distribution to these patients is wide (Fig 1), but most patients experience between 6 and 12 Gy. Unfortunately, the doses of one-fourth of patients exceeded $15 \mathrm{~Gy}$, with few receiving $>25 \mathrm{~Gy}$.

The Joint Commission on the Accreditation of Healthcare Organizations, which monitors hospital practice, currently qualifies single-radiation exposure in excess of 15 Gy to a single field as an "overdose," with potential for substantial harm. ${ }^{25}$ No procedure identified in this study qualified as such a trigger. However, the single-event reporting mandate does not reflect the potential for a cumulative dose during the course of a hospitalization. Because patients who received these doses underwent a mean of 2.8 endovascular interventions and those receiving less radiation underwent a mean of 1.0 interventions, the physician is responsible for monitoring the cumulative radiation dose in the patients who require multiple procedures and are at potential risk for injury.

\section{Mathematic Modeling}

To identify imaging techniques that might reduce patient radiation exposure, dose was recalculated for each patient by using modified imaging techniques (Table). Calculations were made for the following: 1) replacing every portable CT scan with a fixed scan, 2) using the low-radiation acquisition mode of the portable CT, 3) replacing diagnostic angiography with CTA, 4) decreasing fluoroscopy rates from 15 to 10 frames per second, 5) replacing CT with MR imaging, 6 and 7) reducing the radiation dose during DSA from 3.6 $\mu \mathrm{Gy}$ per frame to 2.7 $\mu \mathrm{Gy}$ per frame or $1.8 \mu \mathrm{Gy}$ per frame, and 8 ) a combination of all the above changes. Simultaneous application of all changes included removal of all CT and diagnostic angiography, reducing the fluoroscopy rate to 10 frames per second, and reducing the radiation dose during DSA to $1.8 \mu \mathrm{Gy}$ per frame. Data for these changes were based on medical physics mea- surements as follows: Fluoroscopy at 10 frames per second decreased the dose by $33.3 \%$, and the low-radiation acquisition mode of the portable CT decreased the dose by $50 \%$.

These substitutions or modifications in imaging technique can reduce the cumulative radiation exposure (Table). Substitution of every portable CT scan for a fixed scan reduced exposure by $2.3 \%$. Using the low-radiation acquisition mode of the portable CT reduced exposure by $1.9 \%$. Replacing diagnostic angiography with CTA reduced exposure by $4.9 \%$. Replacing CT with MR imaging reduced exposure by $6.6 \%$. Decreasing fluoroscopy rates from 15 to 10 frames per second reduced exposure by $11.6 \%$. Reducing angiography doses to $2.7 \mu \mathrm{Gy}$ per frame reduced exposure by $13.8 \%$ and to $1.8 \mu \mathrm{Gy}$ per frame reduced exposure by $29.1 \%$. Applying all substitutions reduced exposure by $56.6 \%$.

Incorporating these changes in neuroimaging to reduce the radiation exposure, however, has severe practical limitations. Fixed compared with portable CT scanning reduces radiation exposure but requires patient transport. MR imaging uses no radiation but may be impractical, requiring patient transport often far from patient care floors; is sensitive to patient motion; and is incompatible with certain metallic implants and/or generates potential image artifacts. Lower fluoroscopy rates, lower energy modes of CT scanning, and lower dose angiography do not involve these logistic considerations but trade radiation dose for image quality. The benefits of each imaging modification can be significant but should be carefully weighed against the risks to the patient. However, these suggestions are limited in that the potential dose reduction is at best a calculation and is not very likely exact for each individual situation. Furthermore, radiation-exposure reduction will be most practically and effectively made from the conscientious use of fluoroscopy and angiography.

\section{Physician Awareness}

This study demonstrates the substantial cumulative doses received by patients with subarachnoid hemorrhage during a complete hospital course. Understanding which patients are at even greater risk is important for procedure planning and in medical decisions. Increased length of hospitalization and higher Hunt and Hess grades and Fisher scores are frequently seen in sicker patients with the potential for vasospasm, hydrocephalus, and prolonged intensive care. Although each was anticipated to correlate with radiation dose, this correlation was not demonstrated in this study. The gradual rise in the cumulative dose for Hunt and Hess grades, Fisher scores, and aneurysm number may be suggestive. This effect may have been reduced by the wide variability in radiation exposure and higher mortality more commonly seen in higher grade patients.

The use of endovascular treatment for the primary aneurysm predicted a higher cumulative radiation dose. This result is expected in that the increased exposure is approximately 4 $\mathrm{Gy}$, an average dose reported for a single aneurysm embolization. The decision to clip-versus-coil an aneurysm should not be made with this sole consideration. However, physicians should be aware of this significant contribution to the total radiation exposure.

Interventionalists should be aware that the long-term implications of substantial cranial irradiation may present in a 
delayed manner. ${ }^{21}$ Epilation, skin ulceration, or necrosis and neoplasms may not develop during the initial hospital course and are possible side effects of the radiation doses seen in our patient population. Aggressive screening for signs of radiation-induced injury and incorporation of early management should be part of the routine follow-up examination and care. Additionally, before the procedure, informed consent obtained for the intervention should incorporate the potential high doses of radiation and its associated complications. While mild epilation may be unavoidable and is often reversible, more severe forms of injury are possible and patients should be made aware of this possibility as a component of the preprocedural consent and in postprocedural education and screening.

Because many techniques are available to reduce radiation exposure, proper training highlighting radiation safety can substantially reduce exposure and potential injury to the patient. Maintenance of certification for the use of fluoroscopy equipment should similarly be emphasized to reinforce appropriate practice and encourage radiation-reduction techniques. Hospitals must similarly place particular emphasis on the quality and reliability of neuroimaging equipment. Antiquated fluoroscopy equipment can result in excess radiation through failure in shielding and use of analog rather than digital detection. ${ }^{5}$ The use of updated fluoroscopy equipment and software can minimize the radiation needed to acquire satisfactory images. While each interventionalist should individually decide which imaging changes can be made without compromising the quality of patient care, conscientious use of radiation for diagnostic and therapeutic purposes during prolonged procedures and hospitalizations should be a priority.

\section{Suggestions to Lower the Cumulative Dose}

We demonstrate that patients with subarachnoid hemorrhage can receive significant radiation exposure. Every maneuver that can reduce this exposure should be considered. Because the data demonstrated that most of the exposure is procedural, reducing exposure with appropriate techniques during interventions should be a focus. Such suggestions include reduction in DSA and fluoroscopy time; use of collimators, pulsed fluoroscopy, radiation filters, proper $\mathrm{x}$-ray tube and intensifier placement; and limiting use of electronic magnification and so forth. These techniques should be used when possible.

Furthermore, the application of the recommended techniques to reduce radiation can positively impact this problem. Collimators effectively shield vital structures from direct exposure as well as reduce the tube current necessary to achieve the expected image quality. Using the minimum appropriate magnification reduces the dose but must be balanced against the image detail desired. Whenever anatomically reasonable, using an oblique or lateral imaging path that does not include the orbit can similarly reduce ocular exposure.

\section{Conclusions}

Excessive cumulative radiation doses are possible during the prolonged hospital course of patients with aneurysmal subarachnoid hemorrhage. The reported doses from single inter- ventions can be high but substantially under-represent the cumulative doses seen. Techniques to reduce patient exposure should be applied and conscientious use of radiation for diagnostic and therapeutic purposes during prolonged hospitalizations should be a priority.

\section{References}

1. US Food and Drug Administration. FDA Public Health Advisory: Avoidance of serious X-ray-induced skin injuries to patients during fluoroscopically-guided procedures. http://www.fda.gov/MedicalDevices/Safety/AlertsandNotices/ PublicHealthNotifications/UCM063084. Published September 30, 1994. Accessed March 1, 2001

2. The 2007 Recommendations of the International Commission on Radiological Protection: ICRP publication 103. Ann ICRP 2007;37:1-332

3. Miller L. Interventional fluoroscopy: reducing radiation risks for patients and staff. J Vasc Interv Radiol 2009;20(7 suppl):S274

4. Struelens L, Vanhavere F, Bosmans H, et al. Skin dose measurements on patients for diagnostic and interventional neuroradiology: a multicentre study. Radiat Prot Dosimetry 2005;114:143-46

5. Mooney RB, Flynn PA. A comparison of patient skin doses before and after replacement of a neurointerventional fluoroscopy unit. Clin Radiol 2006;61:436-41

6. Mooney RB, McKinstry CS, Kamel HA. Absorbed dose and deterministic effects to patients from interventional neuroradiology. Br J Radiol 2000;73: $745-51$

7. Theodorakou C, Horrocks JA. A study on radiation doses and irradiated areas in cerebral embolisation. Br J Radiol 2003;76:546-52

8. Gkanatsios NA, Huda W, Peters KR. Adult patient doses in interventional neuroradiology. Med Phys 2002;29:717-23

9. O'Dea TJ, Geise RA, Ritenour ER. The potential for radiation-induced skin damage in interventional neuroradiological procedures: a review of 522 cases using automated dosimetry. Med Phys 1999;26:2027-33

10. Bergeron $P$, Carrier R, Roy D, et al. Radiation doses to patients in neurointerventional procedures. AJNR Am J Neuroradiol 1994;15:1809-12

11. D'Ercole L, Mantovani L, Thyrion FZ, et al. A study on maximum skin dose in cerebral embolization procedures. AJNR Am J Neuroradiol 2007;28:503-07

12. Layton KF, Kallmes DF, Cloft HJ, et al. Radiation exposure to the primary operator during endovascular surgical neuroradiology procedures. AJNR Am J Neuroradiol 2006;27:742-43

13. Bor D, Cekirge S, Turkay T, et al. Patient and staff doses in interventional neuroradiology. Radiat Prot Dosimetry 2005;117:62-68

14. Vano E, Faulkner K. ICRP special radiation protection issues in interventional radiology, digital and cardiac imaging. Radiat Prot Dosimetry 2005;117:13-17

15. Vano E, Gonzalez L, Guilbelalde E, et al. Evaluation of risk in deterministic effects in fluoroscopically guided procedures. Radiat Prot Dosimetry 2005;117:190-94

16. Food and Drug Administration. Performance Standards for Ionizing Radiation Emitting Products, Section 1020.32 Fluoroscopic equipment, and Section 1020.33 Computed tomography (CT) equipment. Silver Spring, MD: Food and Drug Administration, Code of Federal Regulations, Title 21, Volume 8, Part 1020

17. International Commission on Radiation Units and Measurements. Measurement of Dose Equivalents from External Photon and Electron Radiations. Bethesda, MD: International Commission on Radiation Units and Measurements; 1992, ICRU Report 47, Table A.3, page 25

18. Winer-Muram HT, Boone JM, Brown HL, et al. Pulmonary embolism in pregnant patients: fetal radiation dose with helical CT. Radiology 2002;224:487-92

19. Miller DL, Balter S, Cole PE, et al. Radiation doses in interventional radiology procedures: the RAD-IR study. Part II. Skin dose. J Vasc Interv Radiol 2003;14:977-90

20. Wagner LK, Eifel PJ, Geise RA. Potential biological effects following high x-ray dose interventional procedures. J Vasc Interv Radiol 1994;5:71-84

21. Nannapaneni R, Behari S, Mendelow D, et al. Temporary alopecia after subarachnoid haemorrhage. J Clin Neurosci 2007;14:157-61. Epub 2006 Nov 14

22. Huda W, Peters KR. Radiation-induced temporary epilation after a neuroradiologically guided embolization procedure. Radiology 1994;193:642-44

23. Imanishi Y, Fukui A, Niimi H, et al. Radiation-induced temporary hair loss as a radiation damage only occurring in patients who had the combination of MDCT and DSA. Eur Radiol 2005;15:41-46

24. Fletcher DW, Miller DL, Balter S, et al. Comparison of four techniques to estimate radiation dose to skin during angiographic and interventional radiology procedures. J Vasc Interv Radiol 2002;13:391-97

25. Balter S, Miller D. The New Joint Commission sentinel event pertaining to prolonged fluoroscopy. J Am Coll Radiol 2007;4:497-500 